\title{
Review on Maize (Zea mays L.) Breeding Methods for Improving Quality Protein and Others Nutritional Traits
}

\author{
Lemi Yadesa \\ EIAR-Bako National Research Centre, Addis Ababa, Ethiopia \\ Lemi Yadesa, EIAR- Bako National Maize Research Centre, P.O. Box 03, Bako, Ethiopia
}

\begin{abstract}
Maize (Zea mays L.) is a member of the grass family Gramineae (Poaceae) and one of the oldest cultivated crops. To date, maize is widely cultivated in most parts of the world, over a wide range of environmental variables signifying its global and regional importance to millions of people who rely on the crop in pursuit of food security and livelihoods. Now a days, maize mixed with tef is also widely used in towns for making injera. To alleviate malnutrition, protein content can be increased to as high as $18 \%$ by increasing the prolamine (zein) fraction in maize endosperm, but unfortunately it consequently led to lysine and tryptophan deficiency. Since maize is a primary crop in the majority of farming systems and staple food of the rural population in much of the mid-altitude sub- humid agro-ecologies of Ethiopia, substituting the conventional maize (CM) with quality protein maize (QPM) can substantially improve the protein status and greatly reduce the malnutrition problem of resource-poor farmers and low-income people that depend on maize as their staple food. Recent evidence indicates that protein and amino acids play biological roles in protein and lipid synthesis, bone elongation, and the regulation of these and other processes necessary for linear growth. The intensities of lysine or tryptophan should be constantly restrained during the breeding process to sustain the protein quality in grain even when the op2op2 genotype is preserved. The maize kernel, like that of other cereal grains, includes pericarp $(6 \%)$, endosperm (82\%) and germ (12\%).Molecular marker-assisted selection, often simply referred to as markerassisted selection (MAS) offers such a possibility by adopting a wide range of novel approaches to improving the selection strategies in horticultural crop breeding. Appreciative concrete achievements in improving nutritional quality of food crops through conventional breeding efforts necessitate long-term investments, sustained research efforts and patience, besides continuing administrative, financial and scientific support. Further extensive use of MAS is also predictable with the enhancement of methods for marker analysis and identification of applicant genes for commercial traits. Appreciative concrete achievements in improving nutritional quality of food crops through conventional breeding efforts necessitate long-term investments, sustained research efforts and patience, besides continuing administrative, financial and scientific support. Time savings will be attained through MAS may be as significant as cost savings where there are reasonable markets for developed cultivars.
\end{abstract}

DOI: $10.7176 / \mathrm{JBAH} / 11-21-02$

Publication date: November $30^{\text {th }} 2021$

\section{Introduction}

Maize (Zea mays L.) is a member of the grass family Gramineae (Poaceae) and one of the oldest cultivated crops. It is the primary food grain in Mexico, Central America, the Andean region of South America, and eastern and southern Africa. The crop is grown at latitudes varying from the equator to $50^{\circ}$ north and south, from sea level to over 3000 meters elevation, under conditions ranging from heavy rainfall to semi- arid, from cool to very hot climates with growing cycles ranging from 3 to 13 months. To date, maize is widely cultivated in most parts of the world, over a wide range of environmental variables signifying its global and regional importance to millions of people who rely on the crop in pursuit of food security and livelihoods(Demissew Abakemal, 2014).

Maize is a major cereal crop for human nutrition, worldwide. With its high content of carbohydrates, fats, proteins, some of the important vitamins and minerals, maize acquired a well-deserved reputation as a nutriacereal. Several million people, particularly in the developing countries, derive their protein and calorie requirements from maize. It is cultivated on an area of 147 million hectares with production of 692 million tons in the world. India alone accounts for 7.61 million hectares with production of 14.87 million tones where maize follows wheat and rice in importance(Aman, Bantte, Alamerew, \& Tolera, 2016). Maize is a major cereal crop for livestock feed and human consumption and is a raw material for several industrial uses. However, conventional maize has a low protein quality due to its deficiency in two essential amino acids namely, lysine and tryptophan(Jompuk, Cheuchart, Jompuk, \& Apisitwanich, 2011).

Maize has a significant importance in the diets of rural Ethiopia and has gradually penetrated into urban centers. This is particularly evidenced by green maize being sold at road sides throughout the country as a hunger-breaking food. Now a days, maize mixed with tef is also widely used in towns for making injera. Despite its increased consumption, largely as a source of carbohydrates, maize, like all other cereals is known to be of poor protein quality. Protein malnutrition is therefore a problem, especially among children where maize and other cereal crops are the dominant staple foods. Lysine is the first limiting amino acid, followed by tryptophan 
and there online in the diets of non- ruminants and humans. Substituting normal maize with high lysine maize on an equal weight basis can maintain proper amino acid balance(Tulu, Azmach, \& Tesso, 2018a).

A balanced protein is required to assist body building process and therefore, amino acid balance seems to be a determining factor for quality of any food and feed. To alleviate malnutrition, protein content can be increased to as high as $18 \%$ by increasing the prolamine (zein) fraction in maize endosperm, but unfortunately it consequently led to lysine and tryptophan deficiency. Many researchers around the globe have tried to address the problem using quality protein maize (QPM) (Tripathy, Ithape, Maharana, \& Prusty, 2017) .

Efforts to improve the protein quality of maize date back to the $1950 \mathrm{~s}$. In the early $1960 \mathrm{~s}$, the natural o2 mutation was identified as responsible for changing the protein composition of the maize endosperm, nearly doubling its lysine and tryptophan content. As a result, o2 maize grain had improved protein quality, while its protein quantity remained the same. Subsequent conventional plant breeding efforts (i.e., methods not using genetic modification) resulted in agronomically competitive maize varieties adapted to target environments, particularly in sub-Saharan Africa. To differentiate them from the earlier o2 maize varieties and from 'conventional' maize varieties, these new varieties are collectively referred to as quality protein maize (QPM) and are an example of biofortification, or the genetic improvement of the nutritional quality of food crops(Tessema et al., 2016).

Since maize is a primary crop in the majority of farming systems and staple food of the rural population in much of the mid-altitude sub- humid agro-ecologies of Ethiopia, substituting the conventional maize (CM) with quality protein maize (QPM) can substantially improve the protein status and greatly reduce the malnutrition problem of resource-poor farmers and low-income people that depend on maize as their staple food(Tulu et al., 2018a).

\section{Objective of this review}

* To review available information on breeding strategies of maize for Quality protein and other nutrient traits advances.

* To review the highlight of the value of Quality protein for combating malnutrition and food security alleviation.

\section{LITERATURE REVIEW}

\section{The Importance of quality protein Maize}

Maize is one of the most important food crops in the world and together with rice and wheat, providing at least $30 \%$ of the food calories to more than 4.5 billion people in 94 developing countries, which includes 900 million poor consumers. In parts of Africa and Mesoamerica, maize alone contributes over $20 \%$ of food calories. The role of maize for human consumption, expressed in terms of the share of calories from all staple cereals, varies significantly across regions. This ranges from $4 \%$ in South Asia to $29 \%$ in the Andean region to $61 \%$ in Mesoamerica. In sub-Saharan Africa, maize is mainly a food crop accounting for 73 and $64 \%$ of the total demand in eastern and southern Africa (ESA) and West and Central Africa (WCA). This makes maize particularly important to the poor in many developing regions of Africa, Latin America and Asia as a means of overcoming hunger and improving food security(Tuberosa, Frison, \& Graner, 2014).

Africa is a leading continent in the crisis of malnutrition, and the problem of vitamin A deficiency (VAD) this is due to the heavy dependence on white maize in the diet, which causes Kwashiorkor. Vitamin A is a threat to human health, especially in SSA (Sub Sahara Africa) and other developing regions of Asia and Latin America. Among other effects, VAD (Vitamin A deficiency) results in night blindness, loss of appetite, poor growth rate and weakened immune system. Maize consumption in different African countries as a percentage of national diet. Dent, white maize is widely used for human consumption. However, the nutritional composition of white maize lacks provitamin A carotenoids. In plant foods, vitamin A is in the form of provitamin A carotenoids, and crops such as orange maize, orange fleshed sweet potato (Ipomoea batatas L.) that have high content of provitamin A carotenoids are a promising strategy to alleviate vitamin A content among disadvantaged populations(Phakathi, 2015).

Recent evidence indicates that protein and amino acids play biological roles in protein and lipid synthesis, bone elongation, and the regulation of these and other processes necessary for linear growth. Similarly, linear growth is stimulated by insulin-like growth factor, which is also responsive to dietary protein intake. However, dietary intakes of utilizable protein, i.e., protein adjusted for quality (determined by the content of essential amino acids) and digestibility, may be inadequate, particularly in sub-Saharan Africa. Furthermore, current estimates of protein requirements do not address. Children's protein needs for optimal linear growth; increased requirements due to frequent infections, growth faltering, or energy deficit; and the roles of protein and amino acids in growth regulation and immune function. Adjusting protein requirements to account for increased needs due to recurring infections and energy deficits significantly increases estimates of the prevalence of inadequate protein intakes in developing countries(Tessema et al., 2016). 
Growth faltering is widespread among Ethiopian children, and the annual cost of under nutrition to the country has been estimated at US\$4.7 billion, which amounts to $16.5 \%$ of the gross domestic product. Among children under 5 years, $40 \%$ are stunted, $9 \%$ have acute malnutrition, malnutrition, and $25 \%$ are underweight. However, despite these commitments, evidence is still limited, both globally and in Ethiopia, on how agriculture can be effectively leveraged to improve nutrition and health(Tessema et al., 2016).

\section{Definition and Historical Development of Quality Protein Maize}

As researcher reported, the total protein content of maize grain is highly elastic. The most well-known experiment that altered total protein content in maize is the Illinois Long Term Selection Experiment. Starting with a single open pollinated variety, selection for high and low protein has been conducted since 1896. Typical maize lines consist of $8-12 \%$ protein, but selection has resulted in a high protein line with over $32 \%$ protein and a low protein line with $4 \%$ protein. Even though high protein is possible in maize, selection for yield has increased starch and decreased total protein (Bodnar, 2011).

In the maize grain, both the endosperm and embryo contain protein but the embryo protein is superior in quality. However, since the endosperm constitutes the bulk of the grain, it is estimated it may contribute approximately $80 \%$ of the total kernel protein (Eider, 2017). The bulk of maize endosperm protein is comprised of zein which is composed of a class of alcohol soluble proteins that are specific to the maize endosperm. The zeins contain one major class ( $\alpha$-zeins) and three minor classes $(\beta, \gamma, \delta$-zeins). Zeins are the most abundant proteins in the grain endosperm and, particularly, the $\alpha$-zein which are poor in lysine and tryptophan. The ideal approach to improve the nutrition value of maize grain could be suppression of lysine-deficient zein fraction without changing the contribution of other fractions (Tripathy et al., 2017). Quality protein maize (QPM) was developed from mutant maize with an opaque- 2 gene that improves amino acids; lysine and tryptophan. Lysine and tryptophan allow the body to manufacture complete proteins, and tryptophan lessens the prevalence of kwashiorkor in children. Studies clearly show that QPM could be superior to normal maize (NM) if used in the diet of humans to supplement malnutrition(Phakathi et al., 2015).

\section{Nutritional benefits of Quality protein maize}

Maize (Zea mays L.) is one of the most produced crops worldwide. It is ranked in 6th position after rice, wheat, soybean, tomatoes, and sugar cane. Maize is a major staple crop for more than one billion people in sub-Saharan Africa (SSA) and Latin America. In Southern Africa, it is the number one crop which is consumed in not less than two meals a day. Most importantly, maize is not solely used for one objective. It is used for human food, animal feed and industry. Its popularity in SSA is mainly due to its ability to thrive in a wide range of soil types and many agro-ecological zones, and its ability to produce sustainable yields at minimal agronomical inputs for sustainable farmers. However, commonly attained yields are low per unit area. This is not only compromised by poor agronomic practices but also by poor genetic improvements. Thus the production does not match with the human and animal consumption rate (Phakathi et al., 2015).

The term QPM refers to maize genotypes whose lysine and tryptophan levels in the endosperm of the kernels are about twice higher than in CM varieties. Lysine levels in conventional and QPM maize average $2.0 \%$ and $4.0 \%$ of total protein in whole grain flour, respectively. These levels can vary across genetic backgrounds with ranges of $1.6-2.6 \%$ in CM and 2.7-4.5\% in converted QPM counterparts. Despite the nutritional differences, QPM varieties look and perform like CM varieties and one cannot visually distinguish between the two by the physical appearance of the plants or their ears and grains alone. Rather, biochemical analysis is required to determine the lysine and tryptophan content of the seed and confirm whether or not it is QPM(Wegary, Ertiro, \& Bantte, 2015).

Maize is a major cereal crop for both livestock feed and human nutrition, worldwide. With its high content of carbohydrates, fats, proteins, some of the important vitamins and minerals, maize acquired a well-deserved reputation as a 'poor man's nutricerea several million people, particularly in the developing countries, derive their protein and calorie requirements from maize. The maize grain accounts for about 15 to $56 \%$ of the total daily calories in diets of people in about 25 developing countries, particularly in Africa and Latin America1, where animal protein is scarce and expensive and consequently, unavailable to a vast sector of the population. Cereal proteins, however, have poor nutritional value for monogastric animals, including humans, because of reduced content of essential amino acids such as lysine, tryptophan and threonine. Cereal proteins contain on an average about $2 \%$ lysine, which is less than one-half of the concentration recommended for human nutrition by the Food and Agriculture Organization (FAO) of the United Nations.

Therefore, healthy diets for humans and other monogastric animals must include alternate sources of lysine and tryptophan. From the human nutrition viewpoint, lysine is the most important limiting amino acid in the maize endosperm protein, followed by tryptophan. The problem has been mainly dealt by supplementing grain with essential amino acids produced by bacterial fermentation. Although this approach works well for feeding animals, it is highly expensive. Besides, amino acids are often lost from foods processed from grain meals, as in 
the case of maize. For this and other reasons, it is valuable to adopt a genetic enhancement strategy in which essential amino acids are either incorporated or increased in grain proteins. The need to genetically ameliorate the poor nutritional value of cereal grains such as maize has been recognized for a long time. Realizing tangible achievements in improving nutritional quality of food crops through conventional breeding efforts necessitate long-term investments, sustained research efforts and patience, besides continuing administrative, financial and scientific support(Li \& Vasal, 2015a).

The intensities of lysine or tryptophan should be constantly restrained during the breeding process to sustain the protein quality in grain even when the op2op2 genotype is preserved. The consumption of QPM varieties is one way of solving the problem of malnutrition in the world, especially for people poor in resources who cannot access other sources of protein for their families. QPM can also be used as additional food for humans (pregnant women, lactating mothers, and young children, in particular) and to feed animals. Moreover, QPM flour can also be very useful for migrants and other people facing nutritional problems around the world.

\section{Minerals concentration of maize}

The concentration of ash in the maize kernel is about 1.3 percent, only slightly lower than the crude fibre content. Environmental factors probably influence the mineral content. The germ is relatively rich in minerals, with an average value of 11 percent as compared with less than I percent in the endosperm. The germ provides about 78 percent of the whole kernel minerals. The most abundant mineral is phosphorus, found as phytate of potassium and magnesium. All of the phosphorus is found in the embryo, with values in common maize of about 0.90 percent and about 0.92 percent in opaque- 2 maize. As with most cereal grains, maize is low in calcium content and also low in trace minerals. The other fat-soluble vitamin, vitamin $\mathrm{E}$, which is subject to some genetic control, is found mainly in the germ. The source of vitamin E is four tocopherols, of which alpha-tocopherol is the most biologically active. Gamma- tocopherol is probably more active as an antioxidant than alpha tocopherol (Sujatha, 2018).

According to Vasal et al., 2015b reported, in normal maize genotypes, zeins usually account for 50 to $70 \%$ of the endosperm protein and are characterized by a high content of glutamine, leucine and proline. Since zeins are essentially devoid of lysine and tryptophan, they dilute the contribution of these essential amino acids from the other types of endosperm proteins, which are collectively called 'non-zeins'. The non zein fraction contains enzymes, structural polypeptides and membrane-associated proteins. In normal maize, proportions of various endosperm storage protein fractions, on an average, are: albumins (3\%), globulins (3\%), zeins $(60 \%)$ and glutelins (34\%). Significantly, all fractions other than zeins are balanced in amino acid content and are quite rich in lysine and tryptophan. Suppression of lysine-deficient zein fraction without drastically altering the contribution of other fractions could be, thus, seen as a feasible approach to bring about improvements in the amino acid balance in maize grain(Li \& Vasal, 2015b).

Additionally, as researchers reported that, QPM also showed a corresponding increase in tryptophan content, which doubles the biological value of ordinary maize protein. To classify the maize grain as QPM, the quality index, which is the tryptophan-to-protein ratio in a given sample, must be higher or equal to $0.8 \%$. The quality index of hybrids derived from crosses between QPM lines and normal commercial lines was in the range of 0.71 to 0.74 , which was better than in standard hybrids $(0.57-0.62)$ but below the QPM threshold $(0.80)$. Consumption of QPM could go a long way in reducing the growing challenge of malnutrition in various parts of the world. The nutritional benefits of QPM have been demonstrated numerous times in feeding experiments involving livestock and children. For instance, children who ate porridge made from QPM had fewer sick days relative to those who ate porridge from common maize $(\mathrm{CM})(\mathrm{N}$. Tandzi, S. Mutengwa, L. M. Ngonkeu, Woïn, \& Gracen, 2017).

\section{Quality Protein Maize breeding and germplasm development Strategy}

Genetic diversity refers to the variety of alleles and genotypes present in a population. It can be accessed through morphological, physiological, biochemical, and behavioral differences between the individuals within or between the populations. Researchers significantly benefit from the information on genetic diversity of their germplasm. This is because genetic variability is essential to develop a variety. Genetic diversity studies focus on the analysis of variation among individuals or groups of individuals or populations by a specific method or a combination of methods. The data often involve numerical measurements and in many cases, combinations of different types of variables(Phakathi, 2015). Phenotypic expression of germplasm is inherently influenced by their effects of the environment, gene effects and their interaction. The effect of these interactions significantly varies between locations and seasons(Mosisa, Ertiro, Mengesha, \& Keno, 2012).

The maize kernel, like that of other cereal grains, includes pericarp (6\%), endosperm (82\%) and germ $(12 \%)$. The main structural component of the endosperm is starch, a complex carbohydrate that constitutes on an average $71 \%$ of the grain and is a source of concentrated energy. Bulk of the proteins in a mature maize kernel is in the endosperm and germ; but, the germ protein is superior in both quantity and quality(N. Tandzi et al., 2017) 
Quality protein maize looks and performs like CM and one cannot visually distinguish between the two by the physical appearance of the plants or their ears and grains alone. Rather, biochemical analysis is required to determine the lysine and tryptophan content of the seed and confirm whether or not it is. QPM development dates back to the 1920 s when a natural spontaneous mutation of maize with soft and opaque grains was discovered in a maize field in Connecticut, USA.(Tulu, Azmach, \& Tesso, 2018b). The development of inbred lines and identify Protein of their best hybrid combinations are the major focus of maize improvement activities in research program. Large numbers of inbred lines are regularly generated from different sources of germplasm such as local populations, introduced populations, recycled inbred lines and back cross populations, and F2 population of locally adapted hybrids(Mosisa et al., 2012).

QPM development dates back to the 1920s when a natural spontaneous mutation of maize with soft and opaque grains was discovered in a maize field in Connecticut, USA. Two interesting alternatives were considered: (i) exploiting double-mutant combinations; and (ii) simultaneous use of o2 gene and the genetic modifiers of the 02 locus. In several cases, the double-mutant combinations involving 02 and other genes associated with endosperm quality were not always vitreous. Although a double mutant combination involving o2 and sugary-2 (su2) of-fered some advantages such as vitreous kernels, acceptable kernel appearance, lesser ear rot, increased lysine levels and better digestibility of protein, yield was severely affected due to the sum total of independent negative effects of the two mutations. Another double- mutant combination, o 2 fl2, was also researched and not pursued further as modified vitreous kernels were en- countered in only a few genetic backgrounds. The most successful and rewarding option involved a combined use of o2 and the genetic modifiers of the opaque phenotype (Li \& Vasal, 2015a). Numerous exploration revisions have been done over the years to elucidate these constraints on QPM varieties. Different methods have been used so far in the improvement of QPM breeding programs: conventional and molecular approaches.

\section{Quality Protein Maize Development through Conventional Breeding}

According to different research result showed; the opaque-2 allele is recessive in nature, but the endosperm modifiers follow polygenic inheritance. At CIMMYT, a conservative approach is adapted to develop modified opaque $2(\mathrm{mo} 2)$ genotypes to strike a balance between proteins levels and grain quality and competitive yield levels. However, molecular marker based screening for QPM status coupled with phenotypic selection to improve endosperm characteristics seems to be an appropriate strategy for the development of QPM introgression lines. The erstwhile mentioned gene-specific co-dominant markers phi 057(Tripathy et al., 2017). There are various breeding options for developing hard endosperm, high-lysine maize that is competitive in agronomic performance and market acceptance which could be based on specific endosperm high-lysine mutants or other donor stocks. The past approaches involving normal maize breeding populations have centered on altering germ endosperm ratio, selection for multiple aleuronic layers, and recurrent selection to exploit natural variation for high-lysine content. Altering the germ-endosperm ratio to favor selection of larger germ size will have the dual advantage of increasing both protein quantity and quality but it is not practical to attain lysine levels approaching those of 02 maize.

Besides, increased germ size has the disadvantage of contributing to poor shelf life of maize. Recurrent selection for high lysine in normal endosperm breeding populations has been largely unsuccessful due to the narrow genetic variation and heavy dependence on laboratory facilities of this approach. Alternatively, highlysine endosperm mutants provided two attractive options: (1) exploiting double mutants involving 02 and (2) simultaneous use of the 02 gene with endosperm and amino acid modifier genes. In most instances, double mutant combinations involving 02 and other mutants associated with endosperm quality were not vitreous. The most successful and rewarding option exploited combined use ofo 2 with the associated endosperm and amino acid modifier genes.(Tuberosa et al., 2014). Conventional plant breeding is primarily based on phenotypic selection of superior individuals among segregating progenies resulting from hybridization. Although significant strides have been made in crop improvement through phenotypic selections for agronomical important traits, considerable difficulties are often encountered during this process, primarily due to genotype by environment interactions. Besides, testing procedures may be many times difficult, unreliable or expensive due to the nature of the target traits (e.g. abiotic stresses) or the target environment(Lema, 2018).

Segregation and analysis of kernels with a range of endosperm modification began at CIMMYT as early as in 1969 by John Lonnquist and V. L. Asnani as cited in Tuberosa et al., 2014. Initial efforts towards development of QPM donor stocks with good kernel phenotypes as well as good protein quality proved to be highly challenging. Two effective approaches, i.e., intra-population selection for genetic modifiers in o2 backgrounds exhibiting a higher frequency of modified o2 kernels and recombination of superior hard endosperm o2 families, resulted in development of good quality QPM donor stocks with a high degree of endosperm modification. This was followed by the large-scale development of QPM germplasm with a wide range of genetic backgrounds, representing tropical, subtropical and highland maize germplasm and involving different maturities, grain color and texture(Tuberosa et al., 2014). A new variety in conventional breeding could 
take 8 to 10 years to develop. Breeders are very concerned in new technologies to haste up this progression or sort it more effective.

\section{Combining Ability Study to improve maize quality protein traits}

The concept of general combining ability (GCA) and specific combining ability (SCA).GCA refers to the average performances of parents in cross combinations and SCA is the deviation of individual crosses from the average performance of the parents involved. The additive portion of genotypic variance is related to the general combining ability (GCA), determined by mean hybrid performance of a line. The non-additive portions such as dominance and epistasis relate to the specific combining ability (SCA), a measure for cases where some hybrid combinations are better, or worse, than expected based on mean performance of the lines involved. In this design, a set of lines are crossed pair-wise in all possible combinations, providing an assessment of their relative merits to guide selection and testing schemes for the trait under consideration(Demissew Abakemal, 2014).

In breeding, grain yield is the most important quantitative trait which is controlled by multiple genes. The knowledge of the gene actions and their relative contribution in the expression of the trait is very important in breeding process. The non-additive gene actions involved the dominance and epistatic effects of genes for inheritance of the trait. It is difficult to select for yield directly when controlled by non-additive gene action. However, selection for yield under stress conditions can be effectively accomplished through its yield components, which are generally controlled by additive gene action(N. Tandzi et al., 2017). It also enables classification of selected parental materials with respect to breeding behavior in addition to provision of information on the nature of gene action.

\section{Recurrent selection and Back Cross Method to improve quality protein maize}

As the authors reported; recurrent selection is one of the simplest ways to improve a trait, if there is a straightforward way to quantify the trait of interest and a population with heritable variation for the trait. Recurrent selection is simply the selection for a certain trait or traits over many generations, and can be used to control complex multi-genic traits. It results in combinations of genes in a genetic background that directly influence a phenotype(Bodnar, 2011). While nitrogen fertilization is an effective way to increase grain yield of the high protein varieties increasing total protein remains a costly approach to increasing essential amino acid levels. Among all the cereals, maize is the richest source of oil. High oil (HO) maize is an important value-added maize type developed artificially, and a great contribution of modern science to maize breeding. Compared with normal maize, HO maize not only greatly raised the oil content and total energy level, but also increased the protein content, lysine content and other limited amino acidse content. The "added value" of HO maize reflected mainly in the gain-feed-ratio of livestock, swine, and poultry, as well as in the products of maize processing. The development of first HO maize strain (IHO) started in 1896 by Hopkins as cited in was really a historical event which demonstrated the feasibility for altering oil content in maize kernel.(E.O, 2014).

Normal maize carbohydrate consists of about 20.0 - 30.0 per cent amylose and $70.0-80.0$ percent amylopectin. These components vary with variety of seed and environmental conditions. The amylopectin of maize can be used as a substitute of cassava starch in the manufacture of pudding and adhesives while the amylose can be used to form thin, transparent films similar to cellophane and also fibres. An improvement in the chemical composition of maize grain would therefore be of great advantage in the nutritional and industrial uses of the crop. Comstock et al 1994 Obi and Onyishi et al., 1949 cited in (E.O, 2014) proposed reciprocal recurrent selection as one of the most effective breeding methods for developing improved commercial hybrids(E.O, 2014).

Initial QPM breeding efforts at CIMMYT focused on conversion of a range of subtropical and tropical lowland adapted, normal endosperm populations to op2 versions through backcross-recurrent selection procedures, with a focus on accumulating the hard endosperm phenotype, maintaining protein quality, and increasing yield and resistance to ear rot. Basically, two approaches were used in developing QPM donor material: the first approach was intra-population selection for genetic modifiers in opaque-2 backgrounds exhibiting a higher frequency of modified op2 kernels and the second approach involved recombination of superior hard endosperm opaque-2 families. QPM breeding strategies at CIMMYT focus on pedigree breeding whereby the best performing inbred lines, complementary in different traits, are crossed to establish new segregating families. New inbred lines are developed from these segregating families. The opaque-2 mutation is reported to be associated with numerous modifiers which together behave as a polygenic trait. In general, QPM materials had 10-15\% less grain yield with the kernel appearance being dull and chalky, slower drying, as well as the greater kernel rots and greater vulnerability to stored grain pests. Several research studies have been done over the years to solve these constraints on QPM varieties. Different methods have been used so far in the improvement of QPM breeding programs: conventional and molecular approaches.(N. Tandzi et al., 2017). The procedure contains synchronized selection within two genetically variable populations. Each population assists as foundation measureable for selection from the other population. It is therefore the purpose of the current study 
to develop maize populations of increase protein, oil, amylose and amylopectin contents can be used such like technique in order to enhancement of quality of nutritional maize affordably.

\section{Molecular Breeding approaches for Quality Protein Maize development}

The development of molecular markers has led plant breeders to be able to screen large populations of plants for crop improvement. Molecular markers are indicators that can mark specific positions along the genome. However, it is costly and laborious to analyze genetic relationships among populations with large-scale molecular markers. In the same population of plants or on an individual plant the same gene may exist in different alternative forms which are referred to as alleles; thus, molecular markers may be used to identify an allele or allelic combinations expressing a desirable trait. The screening is based on the presence or absence of a certain gene as determined by laboratory procedures, rather than on the visual identification of the expressed trait in the plant(Phakathi, 2015).

Three simple sequence repeat (SSR) markers (phi057, phi112, and umc1066) used in the selection and introgression of the opaque-2 (op2) gene in maize genotypes in Uganda showed that the polymorphic SSR markers (phi057 and phi112) correctly predicted the expression of tryptophan in kernels of all QPM inbreds, as well as five of the six non-QPM inbred lines. These markers could, therefore, constitute the framework for marker assisted introgression of the op2 trait into maize genotypes. One of the main focuses of CIMMYT scientists is to develop reliable, easy-to-use markers for endosperm hardness and free amino acid content in the maize endosperm including high throughput, single seed-based DNA extraction, coupled with low-cost, highdensity SNP genotyping strategies, and breeder-ready markers for some key adaptive traits in QPM maize to enhance efficiency and cost effectiveness of MAS in QPM breeding programs. Proteomic analysis has been also studied in QPM breeding (N. Tandzi et al., 2017). The use of molecular markers in QPM breeding programs reduce the selection process during progress of better-quality genotypes, making it more effective across environments. It is significant to reminder that the innovative generation of markers such as SNPs, are being considered to be relatively more resourceful and inexpensive than older types (SSR, RAPD).

\section{Molecular Basis of QPM O2 (opaque) and Modifier Gene Action.}

The breeding of QPM involves manipulation of three distinct genetic systems (Bjar- nason and Vasal 1992; Krivanek et al. 2007) as cited in (Tuberosa et al., 2014): (1) the recessive mutant allele of the O2 gene, (2) the endosperm hardness modifier genes and (3) the amino acid modifiers/genes influencing free amino acid content in the endosperm. The O2gene was cloned using a transposon tagging strategy with the maize mobile genetic elements, Spm (Schmidt et al. 1990) and Ac (Motto et al. 1988). The O2 gene encodes a leucine-zipper class transcription factor required mainly for the expression of $22 \mathrm{kDa} \alpha$-zein-coding genes and a gene encoding a ribosomal inactivating protein (Lohmer et al. 1991; Bass et al. 1992) as cited in (Tuberosa et al., 2014). Lower $\alpha$ zein content in 02 endosperm results in protein bodies that are about one-fifth to one tenth the normal size, which is presumed to alter packing of starch grains during seed desiccation, thereby conferring a characteristic soft texture to the kernel. With the reduction of $\alpha$-zeins in the endosperm due to 02 mutation, there is a usually concomitant increase in the level of $\gamma$-zeins (Habben et al. 1993) as cited in (Tuberosa et al., 2014).

The homozygous recessive allele causes a decrease of the production of these zeins resulting in a corresponding increase in non-zein proteins, rich in lysine and tryptophan (Gibbon and Larkins 2005) as cited in (Tuberosa et al., 2014). Additionally, the recessive allele of the 02 transcription factor also reduces the production of the enzyme, lysine keto-glutarate reductase, involved in free lysine degradation resulting in enhanced free lysine in the endosperm of 02 maize. In the segregating generations, this recessive allele is selected either visually (identifying mosaic ears on F2 harvests) or using molecular markers. The mechanism(s) by which the endosperm modifier genes convert the starchy endosperm of 02 to a normal phenotype is still not completely understood, but some important clues have been obtained through analysis of biochemical changes in modified o2 endosperm. Studies suggest that the products of the modifier genes interact endosperm hardness modifier genes, which convert the soft/opaque endosperm to a hard/vitreous endosperm without much loss of protein quality, are selected through a low-cost but effective method of light-box screening, where light is projected through the vitreous grains or blocked by the opaque grains.

Endosperm modification is polygenically controlled. However, genetic and molecular analyses revealed some major loci involved in 02 modification; for example, one locus maps near the centromere of chromosome 7 and the second maps near the telomere on the long arm of chromosome 7 (Lopes et al. 1995) as cited in (Tuberosa et al., 2014). Despite the presence of 02 and associated endosperm hardness modifier genes, the lysine and tryptophan levels in segregating families vary widely indicating the existence of third set of genes that modify the amino acid content, which necessitates systematic biochemical evaluation of lysine and/or tryptophan levels in each breeding generation. The lysine content of normal maize is around $2 \%$, whereas it is approximately $4 \%$ (of the total protein) in QPM, with a range $1.6-2.6 \%$ in normal maize and $2.7-4.5 \%$ in QPM. Three genes associated with lysine level have been mapped to locations on chromosome 2, 4 and 7 , besides 
several major o2 modifier-QTLs on chromosomes 1, 7 and 9 (Gibbon and Larkins 2005) as cited in (Tuberosa et al., 2014). Hence, it is conceivable to get promising responses to choice for endosperm texture amendment as well as relative satisfied of the essential amino acids, if they are supervised competently, in the Quality Protein Maize breeding series.

\section{Molecular Marker-Assisted Selection (MAS) in QPM Breeding}

The molecular structure of the opaque- 2 gene was investigated by Henry et al.2005 as cited in Vasal et al., 2015, who found that the molecular diversity in the op2 transcriptional activator was quite high compared to that of other transcription factors in maize. Multiple genes have been identified in controlling amino acid content. At least three loci have been implicated in controlling the levels of a protein synthesis factor correlated with lysine levels and these have been mapped on chromosomes 2, 4, and 7(N. Tandzi et al., 2017).

Molecular marker-assisted selection, often simply referred to as marker-assisted selection (MAS) offers such a possibility by adopting a wide range of novel approaches to improving the selection strategies in horticultural crop breeding. Molecular markers are powerful research tools that make it possible to determine the genetic makeup of plants; they also serve as reference points to compare differences in DNA sequence and consequently, the allele composition between plants. In particular, markers have provided a rapid method to screen parental germplasm for genetic variation, develop genetic linkage maps and tag genes controlling important traits. Both high density maps and markers linked to traits can assist in selecting breeding progeny carrying desirable alleles. Thus, molecular markers bring a systematic basis to traditional breeding, enhancing its precision and expediting the process(Ibitoye, D.O.; Akin-Idowu, 2011).

The 02 gene is recessive and the modifiers are polygenic. Their introgression into elite inbred lines is not straight forward because of three major factors, (1) each conventional backcross generation needs to be selfed to identify the 02 recessive gene and a minimum of six backcross generations are required to recover satisfactory levels of the recurrent parent genome, (2) in addition to maintaining the homozygous o2 gene, multiple endosperm modifiers must also be selected, and (3) rigorous biochemical tests to ensure enhanced lysine and tryptophan levels in the selected materials in each breeding generation require enormous labor, time and financial resources. Although conventional breeding procedures have been used to convert commercial lines to QPM forms, these procedures are tedious and time consuming.

Rapid advances in genomics research and technologies has led to the use of MAS which holds promise in enhancing selection efficiency and expediting the development of new cultivars with higher yield potential. While marker-assisted foreground selection helps in identifying the gene of interest without extensive phenotypic assays, marker-assisted background selection significantly expedites the rate of genetic gain/recovery of recurrent parent genome in a backcross breeding program. With the development and access to reliable PCRbased allele-specific markers such as simple sequence repeats (SSRs) and single nucleotide polymorphisms (SNPs), MAS is becoming an attractive option, particularly for oligogenic traits such as QPM (Tuberosa et al., 2014).

A rapid line conversion strategy for QPM has been developed according to (Babu et al. 2005), cited in (Tuberosa et al., 2014). consisting of a two-generation backcross program that employs foreground selection for the 02 gene in both backcross (BC) generations, background selection at non- target loci in the BC2 generation, and phenotypic selection for kernel modification and other desirable agronomic traits in two subsequent selfed generations. The rapid line conversion strategy outlined in this investigation brings together the salient features of both marker-aided and phenotypic-based selection approaches such as fixing the large segregating generation for the target locus (o2), reducing the linkage drag by selection of flanking markers for recipient allele type, recovering maximum amount of recurrent parent genome within two $\mathrm{BC}$ generations and providing scope for precise phenotypic selection for desirable agronomic and biochemical traits on a reduced number of progeny. The potential benefits of using markers linked to genes of interest in breeding programmes, thus moving from phenotype based towards genotype-based selection, have been obvious for many decades. By now a stage has been reached, where genomics research is focusing on generating functional markers that can help identifying genes that underlie certain traits, thus facilitating their exploitation in crop improvement programs. The mapping of genes controlling agronomic traits coupled with the widespread availability of easy to use simple sequence repeat (SSR) markers and quick DNA extraction methods has provided breeders with an excellent opportunity to apply marker assisted selection (MAS) methods in varies of crops(Lema, 2018).

\section{Marker-assisted pyramiding}

Using MAS, several genes can be combined into a single genotype. Pyramiding is also possible through conventional breeding but it is usually not easy to identify the plants containing more than one gene and phenotypically testing individual plants for all traits can be time-consuming and sometimes very difficult. The most frequent strategy of pyramiding is combining multiple resistance genes. Different resistance genes can be combined in order to develop broad-spectrum and durable resistance to, e.g., diseases and insects. The ability of 
a pathogen to overcome two or more effective genes by mutation is considered much lower compared with the 'conquering' of resistance controlled by a single gene(Lema, 2018). Hence, it may be very challenging to judge plants from certain population types or for traits with damaging bioassays. DNA markers can significantly assist selection since DNA marker evaluates are non-destructive and markers for multiple specific genes can be verified using a particular DNA section without phenotyping.

\section{Marker-assisted backcrossing (MABC)}

Backcrossing is used in plant breeding to transfer (introgress) favorable traits from a donor plant into an elite genotype (recurrent parent). In repeated crossings the original cross is backcrossed with the recurrent parent until most of the genes stemming from the donor are eliminated. However, the donor segments attached to the target allele can remain relatively large, even after many backcrossing generations. In order to minimize this linkage drag, marker assays can be of advantage. Markers can be used in the context of MABC to either control the target gene (foreground selection) or to accelerate the reconstruction of the recurrent parent genotype (background selection). According to Tanksley et al. (1989) cited in (Brumlop \& Finckh, 2011). In traditional backcross breeding the reconstruction of the recurrent parent genotype requires more than six generations, while this may be reduced to only three generations in MABC. These findings are confirmed by results of Frisch et al. (1999) cited in (Brumlop \& Finckh, 2011, who showed in a computer simulation that MAS can reconstruct a level of recurrent parent genome in BC3 which would only be reached in BC7 without the use of markers. However, the authors also state that large numbers of marker data points are required to achieve such results. (Brumlop \& Finckh, 2011).

\section{Marker-assisted recurrent selection (MARS)}

The improvement of complex traits via phenotypic recurrent selection is generally possible, but the long selection cycles impose restrictions on the practicability of this breeding method. With the use of markers, recurrent selection can be accelerated considerably. In continuous nursery programs pre-flowering genotypic information is used for marker assisted selection and controlled pollination. Thus, several selection-cycles are possible within one year, accumulating favorable QTL alleles in the breeding population (Eathington et al. 2007) according to cited in (Brumlop \& Finckh, 2011). Additionally, it is possible today to define an ideal genotype as a pattern of QTLs, all QTLs carrying favorable alleles from various parents. If individuals are crossed based on their molecular marker genotypes, it might be possible to get close to the ideal genotype after several successive generations of crossings. It is likely that through such a MARS breeding scheme higher genetic gain will be achieved than through MABC. Concepts how to achieve the ideal genotype using multitrait selection indices have been developed. Applications in practical plant breeding are not known 4to the authors(Brumlop \& Finckh, 2011).

\section{Seed DNA-Based Genotyping in MAS for Quality Protein Maize Development}

This method is suitable for various types of maize seeds, produces high quality and quantity of DNA and has minimal effects on subsequent germination and establishment. A substantial advantage of this approach is that it can be used to select desirable genotypes before planting, which can bring about dramatic enhancements in efficiency by planting only the plants containing o2 gene in recessive form in $\mathrm{BCnF} 2$ generations of non-QPM $\times$ QPM crosses, and also by minimizing the labor costs and scoring error associated with light box screening of a large number of grains for endosperm hardness. Over several breeding cycles, this is likely to lead to cumulative and accelerated gains in selection pressure (such as light box screening for endosperm hardness modification and systematic biochemical evaluation of lysine and/or tryptophan) and improvements in overall QPM breeding efficiency(Tuberosa et al., 2014).

Many QTL mapping approaches assume that the desirable QTL alleles, once identified, will remain relevant during many cycles of selection, without interactions with other genes or the environment occurring. This is a reasonable objection, considering that QTLs identified in one population do not necessarily show the same effects when introgressed into other genetic backgrounds and that even in constant genetic backgrounds and testing environments QTLs do not necessary. By introgressing three marker-QTL alleles associated with enhanced seedling emergence into elite lines utilizing marker-assisted backcrossing (MABC), this trait was successfully enhanced in sweet corn(Brumlop \& Finckh, 2011).

\section{Transgenic Approach for Different Characters Improved Lysine and Tryptophan Contents.}

The use of molecular biology to isolate, characterize and modify individual genes followed by plant transformation and trait analysis will introduce new traits and more diversity into maize database. For example, maize-based diets (animals or humans) require lysine and tryptophan supplementation for adequate protein synthesis. The development of high lysine maize to use in improved animal feeds illustrates the challenges that continually interlace metabolic engineering projects. From a biochemical standpoint, the metabolic pathway for 
lysine biosynthesis in plants is very similar to that in many bacteria. The key enzymes in the biosynthetic pathway are aspartakinase (AK) and dihydrodipicolinic acid synthase (DHDPS), both of which are feedback inhibited by lysine. Bacterial genes encoding lysine-insensitive forms of aspartakinase (AK) and DHDPS, were isolated from Escherichia coli and Corynebacterium, respectively. Over expression bacterial DHPS in maize seeds overproduced lysine. Likewise, a gene corresponding to resistant form of the enzyme anthranilate synthase (AS) has been cloned from maize and reintroduced via transformation under the control of seed specific promoters. This altered has reduced sensitivity to inhibition by tryptophan, thus overproduced tryptophan.(Sujatha, 2018).

Transgenic strategies can also be used as tools to complement breeding techniques in meeting the estimated levels of provitamin A. In this respect, the over expression of the bacterial genes crtB (for PS) and crtI (for the four desaturation steps of the carotenoid pathway catalyzed by PDS and $\beta$-carotene desaturase in plants), resulted in an increase of total carotenoids of up to 34 -fold with a preferential accumulation of $\beta$-carotene in the maize endosperm. The levels attained approach those estimated to have a significant impact on the nutritional status of target populations in developing countries. Furthermore, the same authors, via gene expression analyses, suggested that increased accumulation of $\beta$-carotene is due to an up-regulation of the endogenous lycopene $\beta$ cylase(Sujatha, 2018).

\section{Genome wide association analysis for carotenoid biofortification in maize}

Along with their essential role in photosynthesis, carotenoids are of significant economic interest as naural pigments and food additives (reviewed in Botella-Pavía \& Rodríguez- Concepción, 2006). Their presence in the human diet provides health benefits as nontoxic precursors of vitamin A and antioxidants, including protection against cancer and other chronic diseases (review by Fraser \& Bramley 2004). These motives have promoted scientists to explore ways to improve carotenoid content and composition in staple crops (reviewed in Sandmann et al. 2006; Zhu et al. 2009).

Analyses of genotypes with yellow to dark orange kernels exhibits considerable natural variation for kernel carotenoids, with some lines accumulating as much as $66 \mu \mathrm{g} / \mathrm{g}$ (e.g. Harjes et al., 2008), with provitamin A activity ( $\beta$ - Icryptoxanthin, $\alpha$ - and $\beta$-carotene is typically small ( $15 \%$ to $18 \%$ of the total carotenoids fraction) compared to lutein or zeaxanthin $(45 \%$ and $35 \%$, respectively; Kurlich \& Juvik, 1999; Brenna and Berardo, 2004). Moreover, a moderate to high heritability estimates indicate that breeding for increased levels of both carotenes and xanthophylls should be feasible(Sujatha, 2018).

Genome-wide association studies (GWAS) have been used extensively to identify allelic variation for genes controlling important agronomic and nutritional traits in plants. Provitamin A (proVA) enhancing alleles of lycopene epsilon cyclase (LCYE) and $\beta$-carotene hydroxylase 1 (CRTRB1), previously identified through candidate-gene based GWAS, are currently used in CIMMYT's maize breeding program. Discovery efforts to understand key genes involved in natural variation for carotenoid content have used genome-wide association (GWAS) approaches to explore allelic variation at loci previously established to be associated with the carotenoid pathway in maize or other model species(Suwarno, Pixley, Palacios-Rojas, Kaeppler, \& Babu, 2015).

\section{Summary}

Generally, the opportunities for applying breeding for improved nutritional quality in crops like maize have increased immensely in the latest years in developed countries. As the application of molecular tools to hurry breeding for enhanced nutritional quality in maize as barely and there is immense prospective and need to increase the opportunity and impact of such operations. Affords an ideal policy upon which a number of nutritionally important characters such as enhanced Fe and $\mathrm{Zn}$, low phytate (for increased bioavailability of nutrients), high provitamin A and high methionine could be combined to descend multiple benefits. Considering the pace of technological developments in genome research, molecular breeding is likely to be the leading option in future for stacking a range of nutritionally important specialty traits.

Appreciative concrete achievements in improving nutritional quality of food crops through conventional breeding efforts necessitate long-term investments, sustained research efforts and patience, besides continuing administrative, financial and scientific support. It is in this context that the story of Quality Protein Maize (QPM) assumes significance, as it not only signifies a breeding achievement of enhancing grain protein quality in maize, but also high- lights the spirit of scientific enquiry through painstaking research, and the ability to pursue 'hunches' against 'odds' through sustained and focused efforts. Further extensive use of MAS is also expectable with the enhancement of methods for marker analysis and identification of applicant genes for commercial traits. The forecasting economics will be a major driver of the application of MAS. For assured traits that are luxurious or logistically difficult to evaluate, MAS is smart substitute. Time savings attained through MAS may be as significant as cost savings where there are reasonable markets for developed cultivars.

\section{For the future prospective Suggestions}


Significant progresses have to be ready, particularly with regard to MAS for generating QPM versions for elite inbred lines in our country to alleviate malnutrition regarding to Maize and other cereal crops and identification of genes/QTLs influencing diverse quality traits in maize also be considered in our national research progressions and highly attained to supply affordable appropriate balanced nutrition to alertly increasing population. As MAS is a methodology that has already substantiated its value in some plant breeding circumstances. It is likely to become more valuable as a larger number of genes are identified and their functions and interactions elucidated. Reduced costs and optimized strategies for integrating MAS with phenotypic selection are needed before the technology can reach its full potential. Generally, marker assisted selection has recognized to be a very valuable technique in plant breeding. Through these techniques, plant breeders have been capable to produce cultivars of agriculturally important plants with genes for enhancing of sustain quality nutrition as well as grain yield at the same time to several nutritional qualities those were not conceivable beforehand the introduction of DNA knowledge.

\section{References}

Aman, J., Bantte, K., Alamerew, S., \& Tolera, B. (2016). Evaluation of Quality Protein Maize ( Zea mays L ) Hybrids at Jimma ,. Forensic Anthropol 2016, 1(1), 1-6.

Bodnar, A. L. (2011). Novel approaches to improving qualities of maize endosperm.

Brumlop, S., \& Finckh, M. R. (2011). Applications and potentials of marker assisted selection ( MAS ) in plant breeding Applications and potentials of marker assisted selection ( MAS ) in plant breeding. In Bundesamt für Naturschutz (BfN),. https://doi.org/10.1016/j.mrrev.2015.03.001

Demissew Abakemal. (2014). Genetic diversity and combining ability of selected quality protein maize (QPM) inbred lines adapted to the highland agro-ecology of Ethiopia. (October), 193.

E.O, O. (2014). Increase In Protein, Oil, Amylose and Amylopectin Contents of Two Populations of Maize (Zea Mays L.) After Two Cycles of Reciprocal Recurrent Selection. IOSR Journal of Agriculture and Veterinary Science, 6(6), 17-22. https://doi.org/10.9790/2380-0661722

Eider, A. (2017). North Dakota State University. Dissertation, (March).

Ibitoye, D.O.; Akin-Idowu, P. E. (2011). Marker-assisted-selection ( MAS ): A fast track to increase genetic gain in horticultural crop breeding. African Journal of Biotechnology, 10(55), 11333-11339. https://doi.org/10.5897/AJB10.302

Jompuk, C., Cheuchart, P., Jompuk, P., \& Apisitwanich, S. (2011). Improved tryptophan content in maize with opaque-2 gene using marker assisted selection (MAS) in backcross and selfing generations. Kasetsart Journal - Natural Science, 45(4), 666-674.

Lema, M. (2018). Marker Assisted Selection in Comparison to Conventional Plant Breeding: Review Article. Agricultural Research \& Technology: Open Access Journal, 14(2). https://doi.org/10.19080/ARTOAJ.2018.14.555914

Li, J. S., \& Vasal, S. K. (2015a). Maize: Quality Protein Maize. Encyclopedia of Food Grains: Second Edition, 4-4(10), 420-424. https://doi.org/10.1016/B978-0-12-394437-5.00223-0

Li, J. S., \& Vasal, S. K. (2015b). Maize: Quality Protein Maize. Encyclopedia of Food Grains: Second Edition, 4-4(November 2001), 420-424. https://doi.org/10.1016/B978-0-12-394437-5.00223-0

Mosisa, W., Ertiro, B. T., Mengesha, W., \& Keno, T. (2012). Genetic Improvement of Maize for Mid-Altitude and Lowland Sub-Humid Genetic Improvement of Maize for Mid-Altitude and Lowland Sub-Humid AgroEcologies of Ethiopia. (January).

N. Tandzi, L., S. Mutengwa, C., L. M. Ngonkeu, E., Woïn, N., \& Gracen, V. (2017). Breeding for Quality Protein Maize (QPM) Varieties: A Review. Agronomy, 7(4), 80. https://doi.org/10.3390/agronomy7040080

Phakathi, L. (2015). Genetic characterization of pro-vitamin A and quality protein maize inbred lines and their derived hybrids degree of Master of Science in Plant Breeding. (August).

Sujatha, M. (2018). Department of Genetics and Plant Breeding, College of Agriculture , ANGRAU, Hyderabad. (March).

Suwarno, W. B., Pixley, K. V., Palacios-Rojas, N., Kaeppler, S. M., \& Babu, R. (2015). Genome-wide association analysis reveals new targets for carotenoid biofortification in maize. Theoretical and Applied Genetics, 128(5), 851-864. https://doi.org/10.1007/s00122-015-2475-3

Tessema, M., Gunaratna, N. S., Donato, K., Cohen, J. L., McConnell, M., Belayneh, D., ... De Groote, H. (2016). Translating the impact of quality protein maize into improved nutritional status for Ethiopian children: study protocol for a randomized controlled trial. BMC Nutrition, 2(1), 1-13. https://doi.org/10.1186/s40795-016-0089-z

Tripathy, S. K., Ithape, D. M., Maharana, M., \& Prusty, A. M. (2017). Quality protein maize (QPM): Genetic basis and breeding perspective. Tropical Plant Research, 4(1), 145-152. https://doi.org/10.22271/tpr.2017.v4.i1.021

Tuberosa, R., Frison, E., \& Graner, A. (2014). Genomics of plant genetic resources. Genomics of Plant Genetic 
Resources, 2(December), 1-515. https://doi.org/10.1007/978-94-007-7575-6

Tulu, D., Azmach, G., \& Tesso, B. (2018a). Perse Performance and Heterosis of Quality Protein Maize (Zea mays L .) Inbred Lines Adapted to Mid-Altitude Sub-Humid Agro-Ecology of Ethiopia. 5(January), 14-26.

Tulu, D., Azmach, G., \& Tesso, B. (2018b). Perse Performance and Heterosis of Quality Protein Maize (Zea mays L .) Inbred Lines Adapted to Mid-Altitude Sub-Humid Agro-Ecology of Ethiopia. (January). 